\title{
The Use of Bromodeoxyuridine-Immunohistochemistry to Identify Transplanted Fetal Brain Tissue
}

\author{
Darlene B. Brown and Brent B. Stanfield \\ Laboratory of Clinical Science, National Institute of Mental Health \\ National Institutes of Health Animal Center \\ Poolesville, MD 20837, U.S.A.
}

\begin{abstract}
The immunohistochemical detection of the thymidine analog, 5-bromo-2'-deoxyuridine (BrdU) is shown to be a useful and reliable method to positively identify fetal brain transplants in standard histological preparations. This technique offers several advantages over the $\left[{ }^{3} \mathrm{H}\right]$ thymidine autoradiographic method, including being much more rapid and avoiding the use of radionuclides.
\end{abstract}

\section{KEY WORDS:}

grafts; cerebral cortex; rats; $\left[{ }^{3} \mathrm{H}\right]$ thymidine; cellular proliferation

While the transplantation of neuronal and non-neuronal tissue into the CNS has become rather commonplace, no generally recognized standards for the histological identification of transplanted tissues have been established. In some instances, of course, the histological verification of the transplant is unnecessary, or, in-

Reprint address:

Brent B. Stanfield

NIMH NIH Animal Center

P.O. Box 289

Poolesville, MD 20837, USA deed, precluded by the experimental design, and in others, localization of the transplant may be possible due to some identifying feature of the transplanted cells, such as a cell-specific, tissuespecific or (in the case of xenografts) strain- or species-specific marker or due to the presence of an introduced marker such as a retrogradely transported fluorescent dye $/ 15,16 /$. Yet in many cases, the identification of transplants has relied solely on their appearance in routine cell-stained preparations - an unreliable procedure for homotopic CNS transplants, especially when fetal brain tissue is transplanted into the developing brain. Some studies have employed the generally applicable technique of autoradiographically identifying donor tissue prelabeled with $\left[{ }^{3} \mathrm{H}\right]$ thymidine $/ 6,7,11,14 /$. Although this method is very reliable and useful it has not been widely adopted, probably because it is expensive and time-consuming and, of course, necessitates all of the precautions attendant to the in vivo use of a radionuclide. During the past several years many studies on the kinetics of cellular proliferation have relied on the use of the thymidine analog, 5-bromo-2'-deoxyuridine (BrdU), which can be detected immunohistochemically /8/. Recently, this method has been shown to be useful for labeling proliferating neuronal populations $19,10 /$. We now report that BrdU can be a useful marker for the unequivocal identification of brain transplants in routine histological sections.

Albino Sprague-Dawley rats obtained from 
Charles River were bred in our colony. The morning when the vaginal wash was found to be sperm-positive was designated embryonic day (E) 0 . On E15 the pregnant rat was given a single intraperitoneal injection of $\mathrm{BrdU}(2 \mathrm{ml} / 100 \mathrm{~g}$ body weight of labeling agent from the Cell Proliferation Kit obtained from Amersham, U.K.;

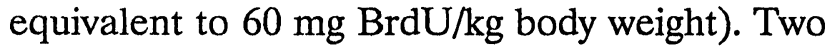
days later the pregnant rat was anesthetized with chloral hydrate $(300 \mathrm{mg} / \mathrm{kg}$ body weight) and the fetuses were removed. The fetal brains were dissected into Ham F10 medium (Biofluids, Inc., Rockville, MD). A piece of either the occipital $(n=4)$ or frontal $(n=-6)$ cortex was dissected from each fetal brain, and trimmed further before transplantation. Newborn (P0) host animals were anesthetized by hypothermia. The scalp was incised and a bone flap made over the occipital cortex. A hole was made in the host occipital cortex by aspiration, the donor tissue was placed into the hole and the bone flap replaced. The scalp incision was closed and the rat pups were allowed to recover from anesthesia on a heating pad before returning to the dam. The host animals were weaned after the third postnatal week and were killed at 33 to 38 days of age. For this, all of the animals were deeply anesthetized with chloral hydrate; four were decapitated and their brains were quickly removed and frozen for cyrostat sectioning; three were perfused through the ascending aorta with $0.9 \%$ $\mathrm{NaCl}$ and their brains were removed and placed into Carnoy's solution for paraffin embedding; and three were perfused through the ascending aorta with $0.9 \% \mathrm{NaCl}$, followed by $10 \%$ formalin in $0.1 \mathrm{M}$ phosphate buffer and their brains were placed in $15 \%$ sucrose in $10 \%$ buffered formalin for frozen sectioning. Some of these animals had 2 to 7 days previously received an injection of a retrograde tracer $(0.2 \mu \mathrm{L}$ of $2 \%$ fast blue or fluoro gold) into the cortex contralateral to the transplant. The brains were processed as recommended in the Amersham Cell Proliferation Kit (Amersham, U.K.), with some modifications. Briefly, the slide-mounted sections were hydrated (paraffin sections were first de-waxed in xylene), treated with $0.07 \mathrm{~N} \mathrm{NaOH}$ for $2 \mathrm{~min}$. and washed in PBS, before being covered with a monoclonal anti-BrdU antibody (Cell Proliferation Kit, Amersham, U.K.) and placed in a humidified box for $40-48$ hours. The sections were then exposed to a peroxidase conjugated anti-mouse IgG and reacted in the presence of hydrogen peroxide and 3,3'-diaminobenzidine tetrahydrochloride, together with cobalt chloride and nickel chloride. Following the peroxidase reaction the sections were dehydrated, defatted in xylene, rehydrated, intensified in $0.005 \%$ $\mathrm{OsO}_{4}$, and coverslipped. An adjacent series of sections was routinely counterstained with thionin, and some of the immunoreacted sections were thionin counterstained before coverslipping.

In the neocortex of the $\mathrm{E} 17$ fetal donor brains large numbers of BrdU-immunoreactive cells are present both in the proliferative matrix lining the lateral ventricles, the ventricular zone $/ 3 /$, and in the cortical plate where they had presumably migrated after their terminal mitosis (Fig. 1). This distribution is essentially identical to that seen at this fetal stage after an E15 injection of $\left[{ }^{3} \mathrm{H}\right]$ thymidine $/ 11 /$. This confirms the previouslv

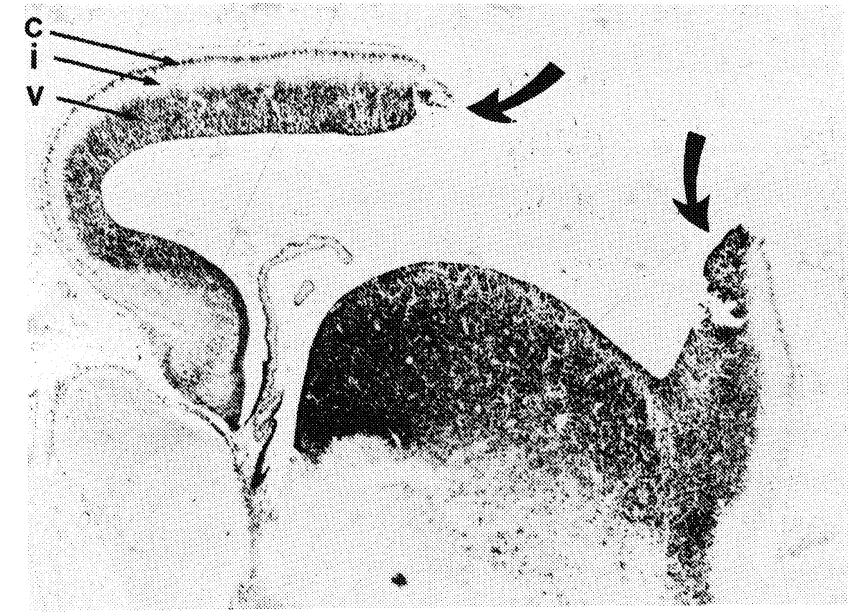

Fig. 1: This is an uncounterstained saggital section through one of the fetal donor brains. Rostral is to the right. The large curved arrows indicate the region of rostral cortex which was removed and further trimmed prior to transplantation. (The host brain into which cortex from this donor was transplanted is shown in Fig. $2 \mathrm{C} \& \mathrm{D}$ ). Note that BrdU-immunoreactive cortical cells are found in the ventricular/subventricular zone ( $V$ ) and in the cortical plate (c), but that few are seen in the intermediate zone (i). Bar $=0.5 \mathrm{~mm}$. 
noted correspondence in both the presence and distribution of labeled cortical cells using $\left[{ }^{3} \mathrm{H}\right]$ thymidine autoradiography or BrdU-immunohistochemistry $/ 9 /$.

In the host animals the clearest delimitation of the transplants is seen in the Carnoy's fixed

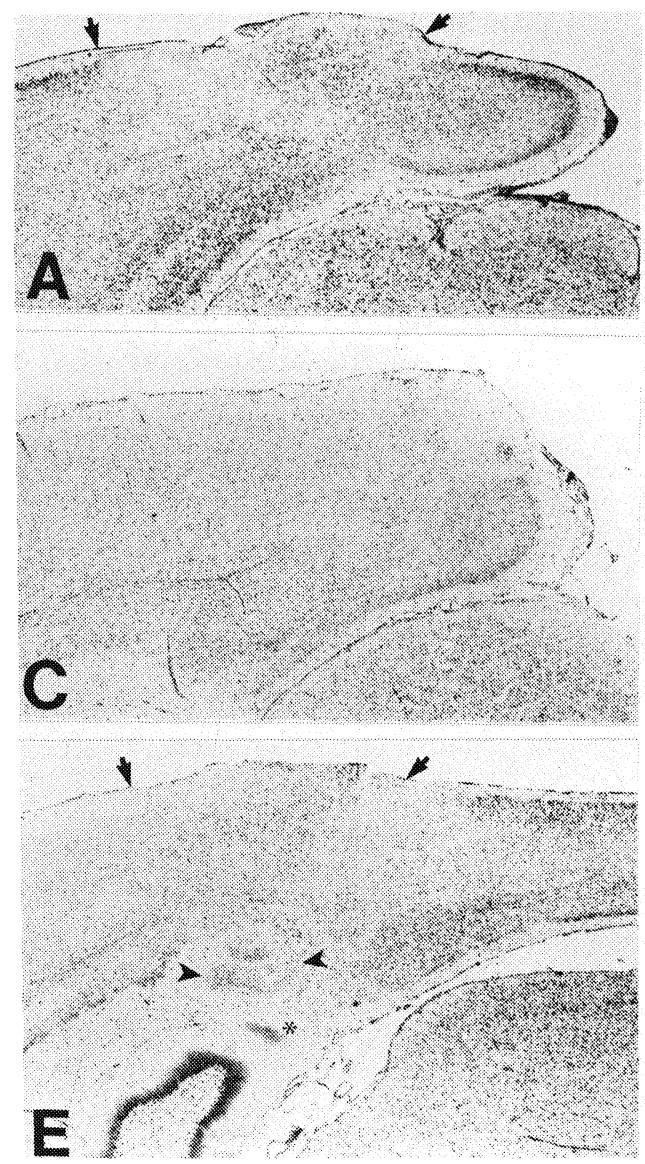

Fig. 2: These photomicrographs are of frontal sections through the occipital cortex from three different transplant cases. Two $10 \mu \mathrm{m}$ thick paraffin sections are shown for each case: one Nissl-stained with thionin (A,C \& E) and an adjacent one processed for BrdU-immunohistochemistry ( $B, D$ \& $F$, respectively). $A$ and $B$ are from a case in which a piece of fetal rostral cortex was transplanted to an occipital locale. In $A$ it can be seen that in the region of the transplant the cortical cytoarchitecture is disrupted and the cell-poor layer I is absent (between the arrows), and the distribution of BrdU-immunoreactive cells in $B$ indicates that this is indeed the transplant. $\mathbf{C}$ and $\mathbf{D}$ are from another rostral-tooccipital cortical transplant case. As is shown in $C$ the Nissl-stained appearance of the cortex suffered only minor disruption in the region of paraffin sections in which optimal immunohistochemistry was obtained. A BrdU-labeled transplant was identified in all four of these animals. Sections from three of these cases are shown in Figure 2. The appearance of the labeled transplants appears comparable to the labeling which
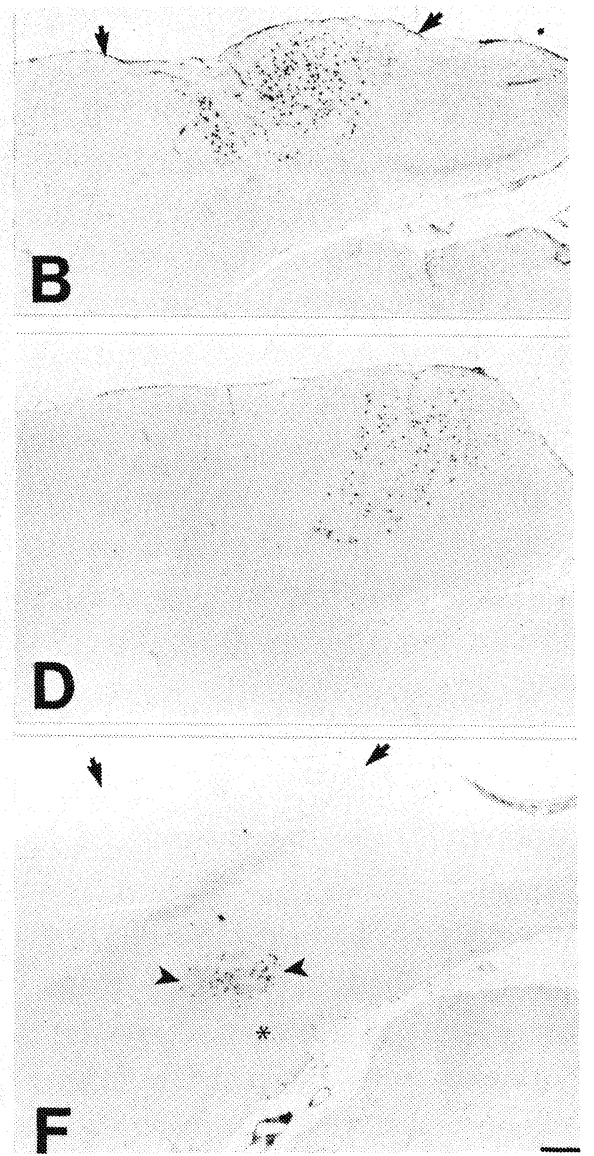

the transplant, and layer I is present, ye " $1 \mathrm{le}$ presence of a wedge-shaped transplant is indicated in the adjacent section shown in D. E and $F$ are from a homotopic transplant case in which a piece of occipital cortex was placed into occipital cortex. There is some disruption of the cortical cytoarchitecture as seen in $\mathrm{E}$, and as in the case shown in A \& B, layer $I$ is absent (between the arrows), yet the distribution of BrdU-immunoreactive cells in the adjacent section $(F)$ indicates that most of the disrupted cortex is host cortex and the transplanted piece of cortex is only the group of cells between the arrowheads. The small clump of cells beneath the transplant $\left(^{*}\right)$ is not immunoreactive, and indeed examination of more rostral sections indicates that this is a piece of the host dentate gyrus granule cell layer. Bar $=0.5 \mathrm{~mm}$. 
is obtained using $\left[{ }^{3} \mathrm{H}\right]$ thymidine as a marker of the transplant $/ 11,14 /$. Certainly the density of BrdU-immunoreactive cells within the transplants is as great, if not greater, than what is found in transplants taken from fetuses exposed to a single dose of $\left[{ }^{3} \mathrm{H}\right]$ thymidine. It should not be surprising that BrdU may provide a more sensitive marker than $\left[{ }^{3} \mathrm{H}\right]$ thymidine, since the short penetrance of the $\beta$-particles emitted by tritium allows the photographic emulsion layer of an autoradiogram to sample only the upper few microns of the section /12/, while BrdU-immunoreactive cells can be labeled throughout even a $30 \mu \mathrm{m}$ thick section $/ 9 /$. As in autoradiograms of $\left[{ }^{3} \mathrm{H}\right]$ thymidine-labeled transplants, the distribution of the BrdU-immunoreactive cells is sometimes skewed, with one part of the transplant having a greater density of labeled cells than another part. As has previously been suggested for $\left[{ }^{3} \mathrm{H}\right]$ thymidine-labeled transplants $/ 11 /$, this distribution of labeled cells may reflect a partial retention of the laminar organization of the transplanted cortex, and hence of the inside-out radial gradient in the time of origin of its constituent neurons $/ 4 /$.

The effectiveness of the BrdU method in providing positive identification of cortical transplants is especially apparent when Nissl-stained sections are compared with adjacent sections processed for BrdU-immunohistochemistry. Often the cellular organization of the cortex as seen in Nissl-stained sections is disrupted in the region of the transplant and the cell-poor layer I may be absent (Fig. 2A,E). Occasionally however, the region of the transplant has a layer I and distortion of the cortex is minimal (Fig. 2C). Adjacent sections processed for BrdU-immunohistochemistry indicate that while the distorted area of cortex which lacks a layer I may correspond to the transplant (Fig. 2B), this is not always the case (Fig. 2F), and that the transplant may, in fact, be present in a region with minimal distortion which may not be apparent in the Nissl-stained section (Fig. 2D).

These observations indicate that Nissl-stained sections alone do not allow for the positive identification of fetal brain tissue transplanted into the developing brain, but that BrdU provides a reliable marker for fetal brain transplants and offers a number of advantages over $\left[{ }^{3} \mathrm{H}\right]$ thymidine autoradiography. First, it takes only a few days to process tissue with the BrdU method, while the exposure time alone for $\left[{ }^{3} \mathrm{H}\right]$ thymidine autoradiography is typically a month or more. Second, the BrdU method avoids all of the precautions and monitoring necessary for the in vivo use of radionuclides. Third, the BrdU method requires no special equipment or facilities other than what is available in a standard histology laboratory, while autoradiography requires a darkroom with controlled temperature and humidity. Fourth, the BrdU method is relatively inexpensive. Finally, a BrdU method kit is commercially available (Cell Proliferation Kit, Amersham, U.K.).

There are, however, some disadvantages to the BrdU method. First, BrdU can be cytotoxic $12 /$, and in some species developing neurons which incorporate BrdU, subsequently die /1,5/. Thus, while BrdU does not appear to be cytotoxic at the doses we have used $/ 9 /$, higher doses or multiple doses of BrdU may affect the viability of the transplant. Second, the efficacy of the method is sensitive to the method of tissue fixation $/ 13 /$. While consistent and reliable results were obtained with Carnoy's fixation, we were unable in our formalin perfused frozen material to produce satisfactory results with the immunohistochemistry while preserving the retrogradely transported dye labeling in the same sections. The method may not then be compatible with certain fixation parameters. But in many instances the BrdU method offers a rapid, reliable and sensitive technique for the definitive identification of transplanted fetal brain tissue.

\section{ACKNOWLEDGEMENTS}

We should like to thank Dr. C. Asanuma for 
her technical advice and for her comments on an earlier version of this manuscript.

\section{REFERENCES}

1. Bannigan JG. Autoradiographic analysis of effects of 5-bromodeoxyuridine on neurogenesis in the chick embryo spinal cord. Dev Brain Res 1987; 36: 161-170.

2. Biggers WJ, Barnes ER, Sanyal MK. Abnormal neural differentiation induced by 5-bromo-2'-deoxyuridine during organogenesis in the rat. Teratology 1987; 35: 63-75.

3. Boulder Committee. Embryonic vertebrate central nervous system: revised terminology. Anat Rec 1970; 166: 257-261.

4. Brückner G, Mares V, Biesold D. Neurogenesis in the visual system of the rat. An autoradiographic investigation. J Comp Neurol 1976; 166: 245-255.

5. Dirbin L, Jacobson M. Effects of 5-bromodeoxyuridine on development of Mauthner's neurone and neural retina in Xenopus laevis embryos. Brain Res 1978; 150: 543-557.

6. Floeter MK, Jones EG. Connections made by transplants to the cerebral cortex of rat brains damaged in utero. J Neurosci 1974; 4: 141-150.

7. Floeter MK, Jones EG. The morphology and phased outgrowth of callosal axons in the fetal rat. Dev Brain Res 1985; 22: 7-18.

8. Gratzner HG. Monoclonal antibody to 5-bromo- and 5-iododeoxyuridine: a new reagent for detection of DNA replication. Science 1982; 218: 474-475.
9. Miller MW, Nowakowski RS. Use of bromodeoxyuridine-immunohistochemistry to examine the proliferation, migration and time of origin of cells in the central nervous system. Brain Res 1988; 457: 4452.

10. Nowakowski RS, Lewin SB, Miller MW. Bromodeoxyuridine immunohistochemical determination of the lengths of the cell cycle and the DNA- synthetic phase for an anatomically defined population. J Neurocytol 1989; 18: 311-318.

11. O'Leary DDM, Stanfield BB. Selective elimination of axons extended by dev eloping cortical neurons is dependent on regional locale: experiments utilizing fetal cortical transplants. J Neurosci 1989; 9: 2230-2246.

12. Rogers AW. Techniques of Autoradiography, 2nd edn. Amsterdam: Elsevier, 1973; 372pp.

13. Scutte B, Reynders MMJ, Bosman FT, Blijham GH. Effect of tissue fixation on anti-bromodeoxyuridine immunohistochemistry. J Histochem Cytochem 1987; 35: 1343-1345.

14. Stanfield BB, O'Leary DDM. Fetal occipital cortical neurons transplanted to the rostral cortex can extend and maintain a pyramidal tract axon. Nature 1985; 313: 135-137.

15. Tanabe T, Ueda S, Sano Y. Combined method of fluorescence tracer technique and PAP immunohistochemistry for discrimination of the transplanted cells. Histochem 1989; 91: 191-194.

16. T $\phi$ nder N, Gaarskjaer FB, Sunde NA, Zimmer J. Neonatal hippocampal neurons, retrogradely labeled with granular blue, survive intracerebral grafting and explantation to tissue culture. Exp Brain Res 1986; 65: 213-218. 

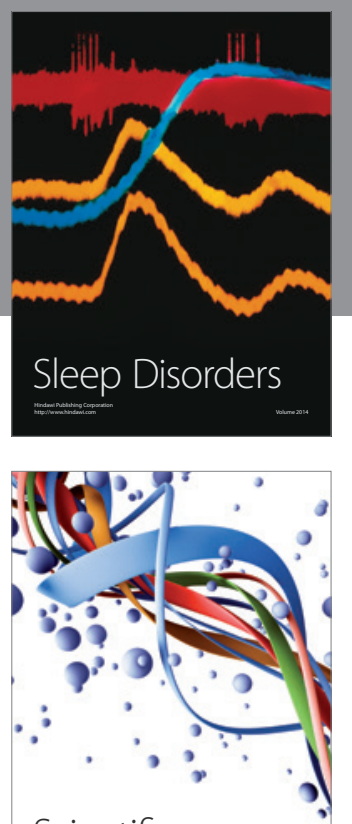

Scientifica
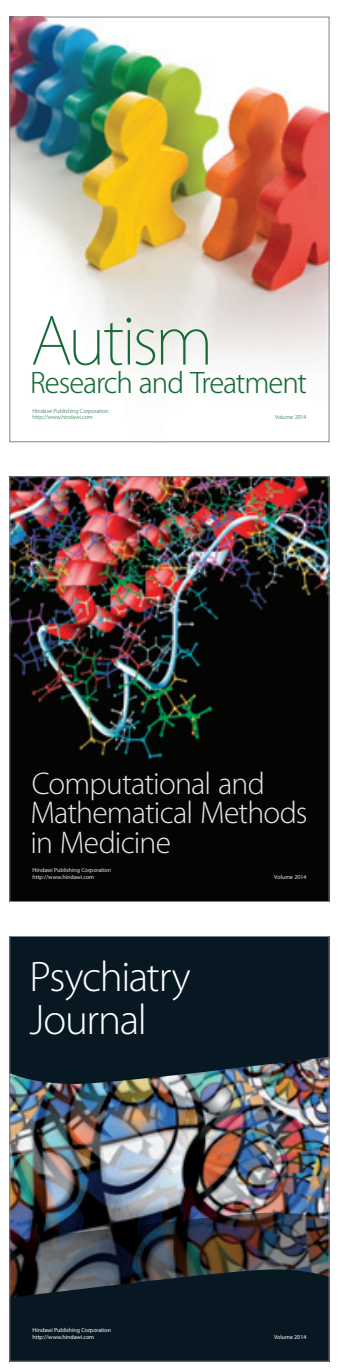
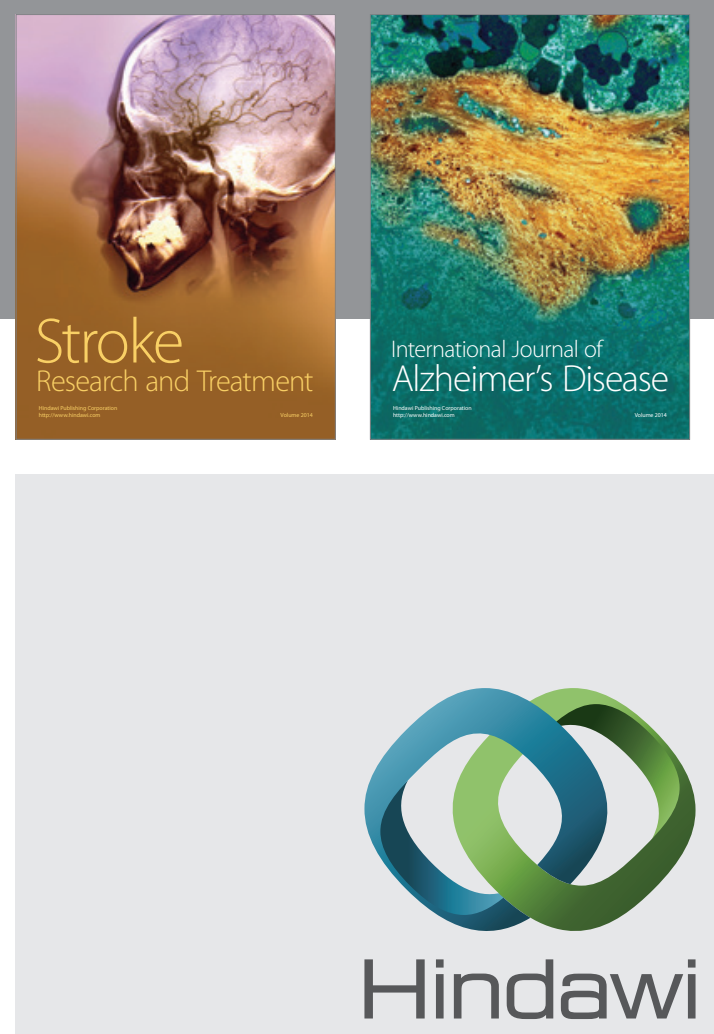

Submit your manuscripts at

http://www.hindawi.com
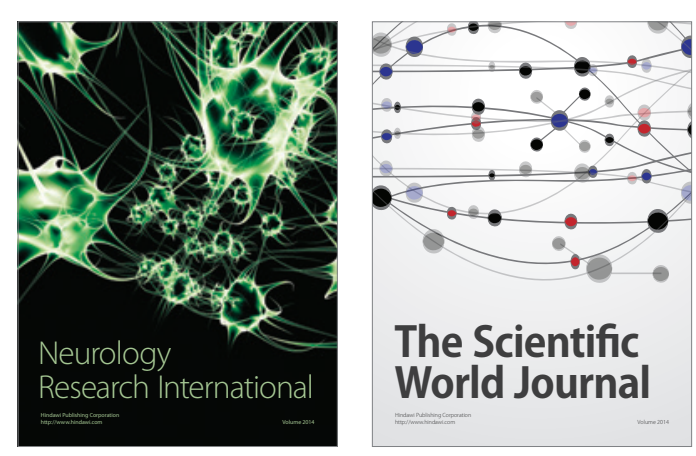

The Scientific World Journal

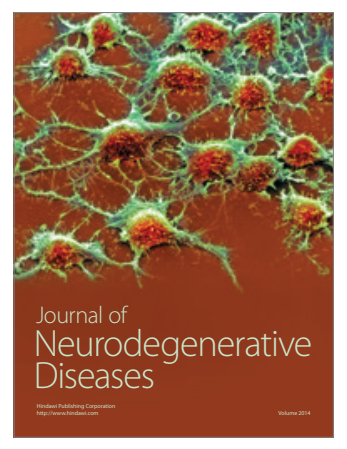

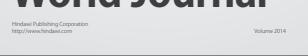

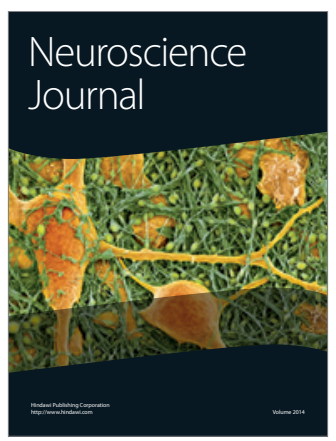

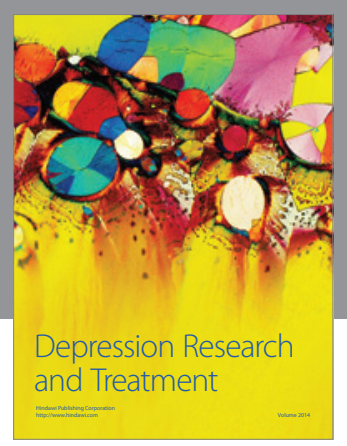
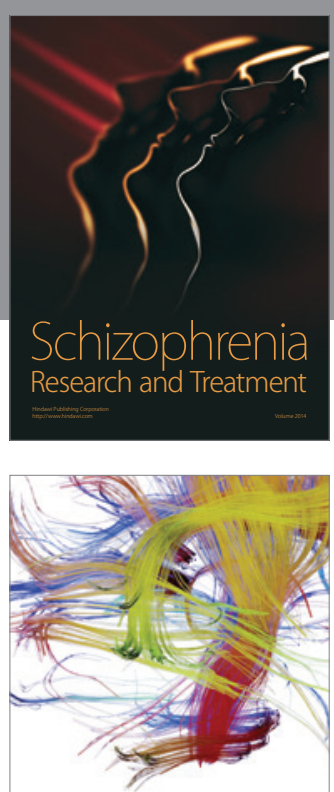

Brain Science

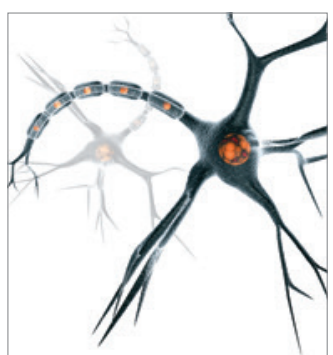

Neural Plasticity
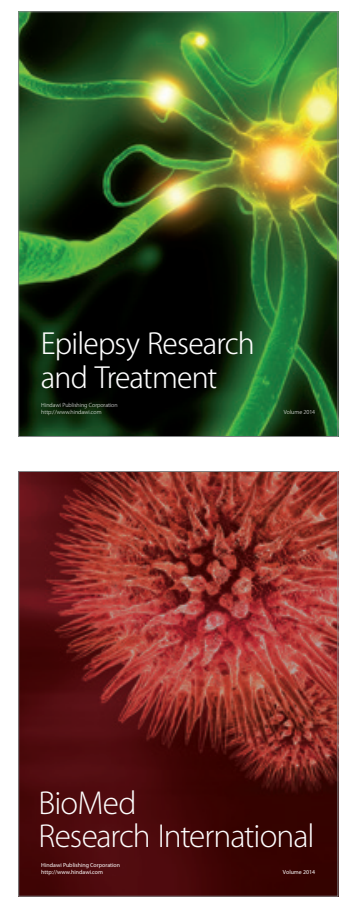

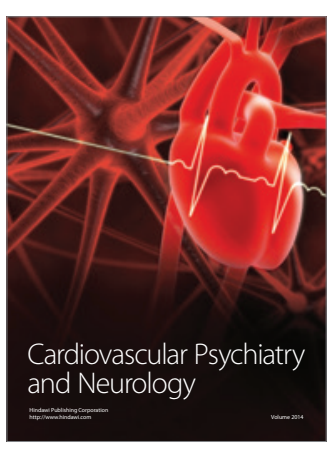

Parkinson's

Disease
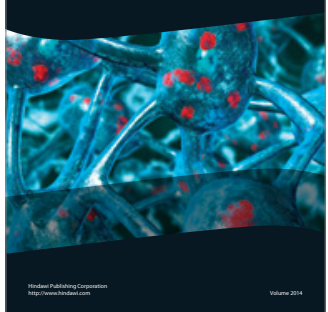